\title{
Redes sociales y protesta política: un análisis del rol moderador del estatus socioeconómico y la pertenencia a grupos políticos
}

\section{Social network sites and political protest: an analysis of the moderating role of socioeconomic status and political group membership}

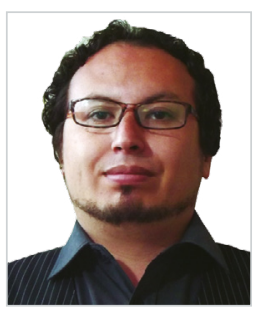

\begin{abstract}
Marcos Zumárraga-Espinosa. Magíster en Gestión Pública por el Instituto de Altos Estudios Nacionales (IAENEcuador) y Economista por la Universidad Central del Ecuador (UCE). Docente investigador de la Universidad Politécnica Salesiana, Ecuador (UPS). Miembro del Grupo de Investigaciones Psicosociales (GIPS-UPS) y del Grupo de Innovación Educativa (GIE-UPS) de Orientación Vocacional y Profesional. Director del proyecto de investigación "Participación política online y offline: relaciones y variables psicosociales" en el cual se enmarca el articulo presentado. Sus principales intereses de investigación son: comportamientos políticos offline y online, rendimiento académico y deserción universitaria, conductas ecológicas y economía conductual.

Universidad Politécnica Salesiana, Ecuador

mzumarraga@ups.edu.ec

ORCID: 0000-0001-9930-9005
\end{abstract}

Recibido: 01/11/2019 - Aceptado: 23/02/2020

\section{Resumen:}

En esta investigación se examina la asociación entre el uso político de redes sociales y la participación en actividades de protesta política a nivel individual, así como el efecto moderador que sobre esta relación ejercen el estatus socioeconómico y la pertenencia a grupos políticos. Para probar las hipótesis planteadas se emplearon datos de encuestas aplicadas en Quito, Ecuador $(N=1520)$ en el año 2018. Los principales resultados son los siguientes: i) el uso de redes sociales (Facebook, Twitter) para el consumo de información y la expresión política influye positivamente sobre el comportamiento de protesta; ii) este efecto movilizador se intensifica en las personas de menor nivel socioeconómico y con mayor grado de participación en agrupaciones políticas offline; iii) la fuerza de las relaciones de moderación descritas se modifica dependiendo de la plataforma digital que se emplee políticamente. Finalmente se discuten las implicaciones de los hallazgos en torno al debate sobre redes sociales, comunicación política y democracia.

Palabras clave:

Participación política; redes sociales; estatus socioeconómico; protesta política; pertenencia a grupos políticos.
Received: 01/11/2019 - Accepted: 23/02/2020

\section{Abstract:}

This research examines the association between the political use of social media and participation in political protest activities at individual level, as well as the moderating effect that socioeconomic status and membership to political groups exert on this relationship. To test the raised hypotheses, the study uses survey data collected in Quito, Ecuador ( $N=1520)$ in 2018. The main results are: (i) the use of social network sites (Facebook, Twitter) for the consumption of political information and political expression positively influences protest behaviour; (ii) this mobilizing effect is intensified in people of lower socio-economic status and with a higher degree of participation in offline political groups; (iii) the strength of the moderation relationships described is modified depending on the digital platform being used politically. Finally, the implications of the findings around the debate on social media, political communication and democracy are discussed.

\section{Keywords:}

Political participation; social media; socio-economic status; political protest; political group membership.

Cómo citar este artículo:

Zumárraga-Espinosa, M. (2020). Redes sociales y protesta política: un análisis del rol moderador del estatus socioeconómico y la pertenencia a grupos políticos. Doxa Comunicación, 30, pp. 55-77. 


\section{Introducción}

El desarrollo democrático de los países se ha caracterizado por la inclusión de la protesta como un componente normal del repertorio de acciones políticas no electorales a disposición de la ciudadanía (DiGrazia, 2014; Schussman y Soule, 2005). Durante la última década, eventos de protesta política ocurridos alrededor del mundo como el movimiento de los indignados y el 15-M en España, el movimiento “Ocuppy Wall Street" en Estados Unidos, las protestas estudiantiles en Chile, \#yosoy132 en México o las recientes manifestaciones de los chalecos amarillos en Francia han demostrado el creciente protagonismo de las redes sociales en la vida política contemporánea. En el plano offline, la protesta política se distingue del activismo convencional por operar al margen de los canales institucionales de participación (Ekman y Amnå, 2012), su naturaleza desafiante con el sistema político (Dalton, 2008), la expresión de inconformidad (Norris, Walgrave y Van Aelst, 2005) y la centralidad del factor organizacional para la movilización efectiva de las acciones colectivas (Anduiza, Cristancho y Sabucedo, 2014). Adicionalmente es frecuente que las acciones de protesta se clasifiquen en blandas y fuertes, según el potencial de violencia y su grado de alejamiento de los límites legales establecidos (Dubrow, Slomczynski y Tomescu-Dubrow, 2008). Considerando estas particularidades, la literatura especializada proporciona cada vez más evidencia empírica compatible con la existencia de una relación entre el uso de redes sociales y el comportamiento de protesta a nivel individual (Boulianne, 2015; Chan, 2016; Cristancho y Anduiza, 2013; Lee, Chen y Chan, 2017; Scherman, Arriagada y Valenzuela, 2015).

De manera más específica, el uso político de plataformas como Facebook o Twitter puede favorecer las dinámicas de protesta política a través de dos mecanismos mutuamente complementarios: el fomento de predisposiciones afines en los usuarios y el fortalecimiento del desempeño organizacional de las agrupaciones de carácter político. El uso de las redes sociales para el consumo y la divulgación de información política, la expresión de opiniones o la implicación en discusiones informales sobre cuestiones de interés público estimula el aprendizaje político en los individuos, lo cual contribuye al reforzamiento del compromiso, las actitudes y el conocimiento político (Eveland, 2001; Eveland, 2004; Lenzi et al., 2015; Pingree, 2007). Por otra parte, las plataformas digitales permiten reducir los costos de organización y coordinación de organizaciones políticas y movimientos sociales, al tiempo que el alcance de sus esfuerzos de reclutamiento se potencia en gran medida (Micó y Casero-Ripollés, 2014; Theocharis et al., 2015).

Investigaciones más profundas se han concentrado en la identificación de los factores que debilitan o intensifican el efecto de las redes sociales, y sus usos políticos, sobre la implicación en actividades políticas offline. Al abordar la protesta política los hallazgos empíricos iniciales indican que el empleo de estas plataformas digitales produce efectos movilizadores diferenciados según el perfil sociodemográfico y actitudinal de los ciudadanos (Valenzuela et al., 2016). A esto se suman las interacciones exploradas entre los predictores individuales del comportamiento de protesta y factores contextuales como el desarrollo económico, la desigualdad social o la madurez democrática (Dalton, Van Sickle y Weldon, 2010). En este sentido, el presente estudio se propone indagar en la relación entre el uso político de redes sociales y la participación en acciones de protesta poniendo énfasis en el rol moderador que pueden ejercer variables individuales como la pertenencia a grupos políticos y el estatus socieconómico. Asimismo, esta investigación se sitúa en el contexto ecuatoriano, mismo que a partir del año 2017 atraviesa un proceso de transición política producto de la finalización del 
periodo de mandato de Rafael Correa (2007-2017). Una década marcada por la baja tolerancia del gobierno frente a la oposición política (Meléndez y Moncagatta, 2017). A pesar de ello, los episodios de protesta social no dejaron de estar presentes, asociándose a cuestiones como la explotación petrolera de una parte del parque nacional Yasuní, las reformas a la educación superior, modificaciones al código penal, entre otras (Basabe-Serrano y Martínez, 2014).

\section{Redes sociales, conductas políticas digitales y protesta política offline}

El entorno digital y las innovaciones en las tecnologías de la comunicación redefinen continuamente los modos de acción política de gobiernos, organizaciones políticas, movimientos sociales y ciudadanía en general. Desde una perspectiva sociológica, la participación política online forma parte de un cambio en la manera de hacer política de las nuevas generaciones, quienes manifiestan mayor preferencia hacia las actividades políticas de carácter expresivo y no convencional (incluyendo actos de protesta), además de vincularse en menor medida con los partidos políticos tradicionales (Calenda y Meijer, 2009). Es así que las redes sociales, y su utilización como herramienta política, han generado efectos beneficiosos tanto en las prácticas habituales de los grupos políticos como en las opciones de participación de los individuos. La esfera digital reduce sustancialmente los costos de comunicación, haciendo posible que las organizaciones políticas y movimientos sociales expandan el alcance de sus acciones en términos de movilización y reclutamiento (Krueger, 2006; Theocharis et al., 2015). Plataformas como Facebook y Twitter abren la posibilidad de difundir mensajes movilizadores a gran escala de forma directa e indirecta (Gibson, 2015; Lee, 2019). Esto último implica dejar que los miembros de las organizaciones asuman un papel más activo y autónomo en la creación, personalización o comunicación de contenidos para movilizar el apoyo de sus contactos, teniendo especial relevancia aquellos vínculos débiles que las redes sociales digitales permiten visibilizar e incrementar (Anduiza et al., 2014; Enjolras, Steen-Johnsen y Wollebæk, 2013; Gustafsson, 2012). Los mensajes difundidos vía redes sociales explican en parte el involucramiento en protestas políticas de aquellas personas que deciden apoyar una causa específica de forma espontánea (Theocharis et al., 2015). Como resultado los grupos políticos son capaces de alcanzar la masa crítica necesaria para coordinar acciones colectivas de manera más rápida y a menor costo gracias a las utilidades brindadas por las redes sociales (Valenzuela, Arriagada y Scherman, 2012).

A nivel individual, se ha encontrado que la frecuencia en el uso de redes sociales se asocia con la participación política offline. Según el meta-análisis desarrollado por Boulianne (2015) a partir de estudios empíricos generados en países desarrollados y en vías de desarrollo, el uso de redes sociales tiende a ejercer efectos significativos sobre la participación política offline, sea de tipo convencional o protesta. Al profundizar en la explicación de dicha relación, los hallazgos de la literatura especializada apuntan hacia la ocurrencia de procesos mediadores como la transición hacia un uso político de estas plataformas digitales, sea por motivos informacionales o expresivos. En otras palabras, a mayor presencia en redes sociales resulta más probable que alguien consuma una mayor cantidad de información política de manera deliberada o incidental (Bode y Dalrymple, 2016; Choi, 2016; Shah et al., 2007; Tang y Lee, 2013). Efecto similar se observa en torno a la expresión de puntos de vista y opiniones políticas (Gil de Zúñiga, Molyneux y Zheng, 2014; Ji, Zhou y Kim, 2017; Shah et al., 2007; Yang y DeHart, 2016). En consecuencia, las conductas políticas informacionales o expresivas desarrolladas mediante espacios como Facebook o Twitter ayudan a fomentar la dimensión política individual, aumentado en conse- 
cuencia la predisposición a participar en actividades políticas offline (Cho et al., 2009; Jung, Kim y Gil de Zúñiga, 2011; Lenzi et al., 2015; Wang, 2007; Yamamoto, Kushin y Dalisay, 2015).

Desde una perspectiva teórica los modelos centrados en el estudio de los efectos comunicacionales plantean que el consumo y divulgación de información de naturaleza política influye positivamente en el comportamiento político de los ciudadanos. Las investigaciones iniciales basadas en el paradigma de los efectos comunicaciones directos plantean que el uso de los medios de comunicación para la búsqueda de información sobre cuestiones de actualidad, temas sociales, políticos o de interés público favorece la implicación en actividades políticas (Gil de Zúñiga, Jung y Valenzuela, 2012; Kaye y Johnson, 2002; Scheufele y Nisbet, 2002). Por el contrario, los usos orientados hacia la diversión o el entretenimiento no presentan efectos significativos (Zhang y Chia, 2006). Estudios subsecuentes han desarrollado modelos de mediación comunicacional para explicar el efecto de los usos informacionales sobre la participación, abordándose recientemente el entorno de los medios sociales. El modelo O-S-R-O-R (Orientación - Estimulo - Razonamiento - Orientación - Respuesta) formulado por Cho et al. (2009) sostiene que el consumo de información política activa procesos autorreflexivos y de procesamiento mental que son elementos necesarios para el posterior involucramiento político. Estos procesos cognitivos a su vez tienden a estimularse gracias a conductas expresivas y la discusión con otros sobre las temáticas revisadas, siendo factores que contribuyen al aprendizaje político. Al respecto, el estudio de Jung et al. (2011) centrado en los usos del internet encontró que la búsqueda y divulgación de noticias duras influye indirectamente en el comportamiento político de los usuarios a través de la expansión de su conocimiento sobre los asuntos públicos, las problemáticas sociales y el proceso político.

Adicionalmente los esfuerzos de composición que demanda la publicación de opiniones, al igual que el componente deliberativo de las discusiones que pueden desencadenarse posteriormente, son elementos que también aportan al fortalecimiento del compromiso y el conocimiento político. Para Pingree (2007) la expresión política está compuesta por tres mecanismos subyacentes que impactan en el emisor: la expectativa previa a la expresión, la composición del mensaje y los efectos de su difusión. En conjunto estas etapas motivan la reflexión crítica, el aprendizaje de nuevos contenidos, la elaboración mental y el compromiso con las opiniones vertidas. En este punto, las redes sociales generan condiciones favorables en términos de costos, alcance e inmediatez para el desarrollo de conductas expresivas. Partiendo de los supuestos del modelo teórico de las ganancias diferenciales, la variedad de actividades expresivas que facilitan las redes sociales estimulan el aprendizaje político significativo (Yamamoto et al., 2015). Dicho efecto se debe no solo a que los contextos comunicativos interpersonales promueven la toma de mayor consciencia e interés sobre los acontecimientos actuales, las cuestiones sociales y los contenidos políticos discutidos, sino que también motivan a los usuarios a buscar información adicional y sopesar diferentes perspectivas con el objetivo de formular argumentos de mayor calidad, anticipando los desacuerdos que pudieran surgir (Eveland, 2004; Pingree, 2007; Shah et al., 2005). De modo complementario el aprendizaje político influye positivamente en los sentimientos individuales de autoeficacia política, lo cual a su vez estimula las conductas participativas (Jung et al., 2011; Heiss y Matthes, 2016).

En resumen, se espera que los procesos señalados eleven la predisposición de los usuarios para comportarse políticamente. Investigaciones previas presentan entre sus hallazgos efectos positivos de parte de los usos informacionales y expresivos de las redes sociales sobre la participación política offline convencional y de protesta (Conroy, Feezell y Guerrero, 
2012; Gil de Zúñiga, Ardèvol-Abreu y Casero-Ripollés, 2019; Macafee y De Simone, 2012; Yamamoto et al., 2015; Yoo y Gil de Zúñiga, 2014; Zumárraga-Espinosa, Reyes-Valenzuela y Carofilis-Cedeño, 2017).

\section{El rol moderador de la pertenencia a grupos políticos y el estatus socioeconómico}

La literatura proporciona cada vez más conocimiento sobre los procesos mediadores que explican el impacto de las redes sociales en la participación política online y offline. Sin embargo, queda por precisar los factores que pueden condicionar la intensidad de los efectos movilizadores producidos por el uso político de estas plataformas. Investigaciones empíricas muestran que variables individuales como los rasgos de personalidad, la educación cívica o los valores políticos interactúan con la relación entre el uso general de redes sociales y el comportamiento político convencional en el ámbito offline (Kim, Hsu y Gil de Zúñiga, 2013; Xenos, Vromen y Loader, 2014). Esta relación también tiende a condicionarse por propiedades de la red social digital del usuario, como su tamaño o heterogeneidad (Campbell y Kwak, 2011). Al abordar el uso político de las plataformas digitales se ha podido observar que el uso de tipo expresivo tiende a incrementar el efecto de los usos informacionales (leer noticias sobre temas políticos o sociales, visitar foros online o compartir contenidos políticos, entre otros) sobre la participación política offline (Yamamoto et al., 2015). El referido efecto interacción se ha explicado teóricamente a partir de modelo de ganancias diferenciales (Hardy y Scheufele, 2005). En el caso de la protesta política, los hallazgos realizados por Valenzuela et al. (2016) a partir de datos de diecisiete países latinoamericanos sugieren que aspectos como el género, la edad, la ideología política y la interacción con organizaciones sociales moderan la relación entre el uso político informacional de redes sociales (Facebook, Twittery Orkut) y la participación en acciones de protesta. También se ha observado que la inclinación hacia valores postmaterialistas en términos culturales no constituye un moderador significativo (Valenzuela et al., 2012).

Según el modelo de voluntarismo cívico propuesto por Verba, Schlozman y Brady (1995) la disponibilidad de recursos cognitivos y materiales (dinero, tiempo y habilidades cívicas), al igual que los vínculos con asociaciones de naturaleza política son determinantes importantes de la participación política offline. No obstante, existe la posibilidad de que estos factores también condicionen el impulso movilizador del uso político de redes sociales sobre la participación offline, incluyendo los comportamientos de protesta. La incorporación de la esfera digital a la vida política ha estado acompañada de visiones optimistas y pesimistas con respecto al potencial del internet para el fortalecimiento de la democracia. Desde una perspectiva optimista el entorno online ofrece una alternativa menos costosa para la participación en actividades políticas. De esta manera se facilita la movilización política de aquellas personas que han permanecido inactivas en el plano offline por sus limitaciones de recursos (Krueger, 2002). Por el contrario, la perspectiva pesimista propone que el internet y los avances en las tecnologías de la comunicación tienden a ser aprovechados por las mismas personas que se encuentran activas políticamente de manera offline. En otras palabras, los recursos disponibles siguen jugando un papel decisivo al momento de aprovechar las oportunidades brindadas por la esfera digital en términos de acción política (Schlozman, Verba y Brady, 2010).

Varias investigaciones se han propuesto verificar estas tesis contrapuestas, encontrándose en algunos casos que si bien aspectos como la educación y el nivel de ingresos siguen influyendo positivamente en la participación política online, su 
efecto tiende a ser menor al ejercido sobre el activismo offline (Gil de Zúñiga et al., 2012; Winneg, 2009). Una vez se cuenta con acceso al internet los recursos dejan de limitar las conductas políticas digitales (Anduiza, Gallego y Cantijoch, 2010). Asimismo, se ha observado el surgimiento de un sector de la población que siendo inactivo en el plano offline reporta comportamientos políticos en la esfera online (Nam, 2012). Otros estudios incluso han detectado que los individuos de menor estatus socioeconómico tienden a reportar mayor participación online (Krueger, 2002; Gil de Zúñiga et al., 2010; Yoo y Gil de Zúñiga, 2019; Zumárraga-Espinosa, Carofilis-Cedeño y Reyes-Valenzuela, 2017).

Los hallazgos efectuados sugieren que el internet y las redes sociales podrían contribuir a una mayor democratización de la participación política entre los miembros de la sociedad. A propósito de esto, la investigación empírica desarrollada por Morris y Morris (2013) muestra que el uso del internet impacta con mayor fuerza en el comportamiento político de quienes pertenecen a los estratos socioeconómicos más bajos. Una explicación tentativa propone que las personas con menor educación e ingresos obtienen mayores beneficios en términos de aprendizaje y consolidación de actitudes políticas gracias a las facilidades otorgadas por el internet. Esto adquiere sentido dado que el espacio digital reduce sustancialmente las dificultades que los grupos menos favorecidos afrontaban tradicionalmente al momento de acceder a información y noticias sobre los asuntos públicos, contar con oportunidades para la expresión de puntos de vista y la discusión política, o entrar en contacto con organizaciones de naturaleza política (Carlisle y Patton, 2013; Oser, Hooghe y Marien, 2013). Cabe señalar que aunque toda la población se beneficia con la reducción de costos de la participación política en su modalidad online, son las personas de menor estatus socioeconómico quienes experimentan cambios más importantes en torno a su posibilidad de desarrollar las conductas arriba mencionadas. Por el contrario, los sectores sociales más favorecidos han gozado de mayores oportunidades para expresarse, informarse y mantener conexiones con actores o asociaciones políticas desde el periodo pre-internet (Best y Krueger, 2005; Brady, Verba y Schlozman, 1995).

A esto debe sumarse la capacidad de las redes sociales para estimular conductas políticas digitales en personas con ingresos más bajos, menor educación y poco interesadas, poniendo en marcha mecanismos como la exposición incidental a información política y estímulos movilizadores (Kim, Chen y Gil de Zúñiga, 2013; Gustafsson, 2012; Valenzuela, 2013), disponibilidad de audiencias permanentes para expresión de la voz política individual (Gil de Zúñiga et al., 2014) y expansión del capital social en entornos interactivos (Gil de Zúñiga et al., 2012; Valenzuela, Park y Kee, 2009). Lo expuesto coincide en parte con la investigación efectuada por Valeriani y Vaccari (2016) a partir de muestras representativas de distintos países europeos, donde se encontró que la exposición incidental a información política vía medios sociales se relaciona más intensamente con la participación política online cuando se trata de personas que reportaron menor interés en la política. Este razonamiento sugiere que los usos informacionales y expresivos de plataformas como Facebook o Twitter pueden producir ganancias diferenciales en términos cognitivos y actitudinales, beneficiando en mayor medida a las personas de menor estatus socioeconómico. Así, dichas ganancias diferenciales incrementan con mayor fuerza la predisposición a participar políticamente de los grupos sociales menos favorecidos. De ocurrir tal efecto democratizador, se esperaría que el uso político de redes sociales estimule con mayor intensidad la participación política offline en aquellos ciudadanos de menor estatus socioeconómico. Esto aplicaría también para las conductas de protesta política.

Por otra parte, los grupos políticos constituyen espacios propicios para el cultivo de vínculos interpersonales y el contacto con otras organizaciones que comparten intereses comunes (Schussman y Soule, 2005). Con su llegada, el internet 
y las redes sociales no solo han reducido drásticamente los costos comunicacionales de las organizaciones políticas y movimientos sociales, también han aportado un carácter más descentralizado a su funcionamiento, creando oportunidades para que sus miembros adopten un rol más activo y autónomo en la promoción de los objetivos organizacionales (Gibson, 2015; Theocharis et al., 2015). Tales condiciones podrían intensificar los efectos positivos a nivel cognitivo y actitudinal que genera el uso político de plataformas como Facebook o Twitter. En primer lugar, el consumo de información política vía redes sociales adquiere mayor calidad movilizadora para quienes pertenecen a agrupaciones políticas offline. Esto ocurre porque las agrupaciones políticas operan como redes de movilización a través de los cuales circula información directamente vinculada con el desarrollo de iniciativas y actividades políticas en el mundo real, tal es el caso de invitaciones, convocatorias, propuestas de acción, lugares de encuentro, agentas activistas, entre otras (Best y Krueger, 2005; Carty, 2010; Lemert, 1981; Micó y Casero-Ripollés, 2014). Dadas estas condiciones, el uso informacional de redes sociales de quienes pertenecen a grupos políticos cuenta con una mayor carga de información movilizadora.

En segundo lugar, los grupos políticos offline poseen una serie de características que favorecen los procesos cognitivos y actitudinales asociados tanto con la expresión como la discusión política. Si bien la relación entre pertenencia a organizaciones de la sociedad civil y capital social se ha venido estudiando desde hace mucho tiempo (Putnam 2000), Conroy et al. (2012) resaltan que los grupos políticos promueven la participación política a través de dos mecanismos: a) la creación de condiciones estimulantes para la discusión política y b) el control mutuo de las actividades y aportes de los miembros. No obstante, la interacción que toma lugar en redes sociales solo reproduce adecuadamente el primer mecanismo, mientras que las posibilidades de control sufren limitaciones debido a la falta de contacto cara a cara. En este sentido, la expresión digital de opiniones tiene más probabilidades de retroalimentación gracias a las contribuciones de los demás miembros de la organización, quienes comparten interés por la política a diferencia del resto de la lista contactos.

Esto eleva la propensión a participar en discusiones electrónicas sobre cuestiones políticas para quien pertenece a grupos políticos offline, más aun si estos grupos están presentes en redes sociales. Por lo tanto, la pertenencia a grupos políticos offline asegura en mayor medida que los usos informacionales y expresivos de las redes sociales generen retornos favorables en términos de aprendizaje político, compromiso cívico e identidad de grupo. Aspectos de vital importancia para la gestación de acciones colectivas, particularmente necesarias para la protesta política (Schussman y Soule, 2005; Valenzuela et al., 2012).

Adicionalmente, pese a que los usuarios se encuentran más inclinados a incluir en su lista de contactos a personas que piensan de manera similar, lo cual se ha definido como selectividad social (Campbell y Kwak, 2011), los grupos políticos offline limitan en cierta medida esta posibilidad. Aunque en términos generales quienes integran una organización política o movimiento social comparten intereses comunes, eso no implica que puedan existir puntos de vista y opiniones heterogéneas al abordarse temáticas más específicas. Al respecto, estudios han mostrado que la calidad deliberativa de la discusión política aumenta cuando existe heterogeneidad de puntos de vista, lo cual a su vez mejora las ganancias de conocimiento político (Mutz, 2002). Por último, es probable que los mensajes políticos generados por miembros de organizaciones mediante redes sociales cuenten con una mayor carga movilizadora. Como se había mencionado, las redes sociales permiten que los miembros de grupos políticos se involucren más activa y autónomamente en el impulso de las agendas organizacionales. Es así que los miembros disponen de mayores incentivos para generar estímulos movilizado- 
res a partir del uso político de sus cuentas de Facebook o Twitter. Las acciones movilizadores pueden dirigirse hacia otros miembros a modo de mensajes que propongan, convoquen o promuevan la acción, o hacia usuarios no militantes con fines de reclutamiento (Gibson, 2015; Gustafsson, 2012). Según los hallazgos de Rojas y Puig-i-Abril (2009) las personas que desarrollan esfuerzos de movilización hacia otros se encuentran más predispuestas a enrolarse en actividades de participación política offline. En resumen, la suma de los procesos descritos sugiere un reforzamiento de la conexión entre los comportamientos políticos vía redes sociales y la participación en actividades de protesta para quienes se encuentran vinculados con agrupaciones políticas.

La discusión efectuada a partir de la integración de contribuciones teóricas y empíricas provenientes de la literatura especializada permite fundamentar las siguientes hipótesis de investigación:

Hipótesis 1 (H1): El uso político de redes sociales se relaciona positivamente con el comportamiento de protesta política.

Hipótesis 2 (H2): La relación entre el uso político de redes sociales y el comportamiento de protesta política se encuentra moderada por la pertenencia a grupos políticos.

Hipótesis 3 (H3): La relación entre el uso político de redes sociales y el comportamiento de protesta política se encuentra moderada por el estatus socioeconómico.

Con el propósito de realizar una prueba conservadora de las relaciones planteadas se controlará el efecto de un conjunto de predictores que están conceptualmente relacionados con la protesta política. Partiendo de las teorías explicativas disponibles en la literatura es posible agrupar a los determinantes individuales del comportamiento de protesta en cuatro grandes categorías: inconformidad, limitaciones y recursos individuales, actitudes políticas y oportunidades de reclutamiento (Dalton, Van Sickle y Weldon, 2010; DiGrazia, 2014; Schussman y Soule, 2005). En el caso de la inconformidad con el desempeño del gobierno y las problemáticas sociales se incluyen variables como la eficacia política externa y las emociones negativas con respecto a la situación social y económica del país. Las limitaciones individuales hacen referencia a aquellos factores capaces de incrementar el costo y los riesgos de participar en acciones de protesta, razón por la cual actúan como restricciones para este tipo de comportamientos. En esta categoría se contemplan las características sociodemográficas y la situación ocupacional de los ciudadanos. Asimismo se requiere de disponibilidad de recursos materiales y cognitivos para afrontar los costos de participar políticamente. Para este punto se considera la variable estatus socioeconómico. Entre las actitudes políticas consultadas se encuentra la eficacia política interna, el interés por la política y la ideología política. Se incluye el consumo de medios de comunicación tradicionales como un factor capaz de alterar la actitud de los usuarios hacia los movimientos de protesta (Boyle y Schmierbach, 2009). Finalmente, la pertenencia a grupos políticos actúa como indicador de contacto y exposición a los esfuerzos de reclutamiento de organizaciones políticas o movimientos sociales. 


\section{Método}

\subsection{Muestra y procedimiento}

Esta investigación empleó datos de una muestra de 1520 participantes radicados en el Distrito Metropolitano de Quito (DMQ), Ecuador. Se efectuó un muestreo no probabilístico, sin embargo se introdujeron cuotas por género y administración zonal para aumentar la representatividad de la población quiteña en términos demográficos y geográficos. En el estudio participaron 765 hombres (50.3\%) y 755 mujeres (49.7\%). El 55.7\% de la muestra reportó edades de 18 a 29 años, $25.7 \%$ de 30 a 44 años, $16.6 \%$ de 45 a 64 años y 2\% de 65 años en adelante. La edad promedio fue de 31.9 años (DT=13.0). El nivel de educación de los participantes se distribuyó de la siguiente manera: $0.3 \%$ sin instrucción formal, $0.7 \%$ primaria incompleta, $3.3 \%$ primaria completa, $5.4 \%$ secundaria incompleta, $5.1 \%$ secundaria completa, $4.5 \%$ bachillerato incompleto, $25.3 \%$ bachillerato completo, 30.7 universidad incompleta, $21.2 \%$ universidad completa y el $3.5 \%$ cuenta con estudios de posgrado. El ingreso familiar mensual se registró a través de una escala con intervalos que van desde "385 USD o menos" (19.9\%) hasta “3474 USD o más" (2.8\%), la moda se situó en el segmento de "386 hasta 771 USD” (27.4\%). Los datos provienen de la Encuesta General de Opinión y Participación Política desarrollada y aplicada periódicamente por el Grupo de Investigaciones Psicosociales de la Universidad Politécnica Salesiana (GIPS-UPS). La recolección de información tuvo lugar entre los meses de junio y julio del 2018 con la ayuda de estudiantes universitarios. Previo a la aplicación del instrumento, los participantes fueron informados sobre los protocolos de confidencialidad y los objetivos del estudio.

\subsection{Variables}

Comportamiento de protesta: Se evaluó a partir de 6 ítems correspondientes a las siguientes actividades de protesta política: participar en huelgas o manifestaciones no autorizadas, tomar parte en protestas colectivas, rehusar pagar impuestos, bloquear el tráfico, participar en la ocupación de edificios públicos, boicotear o deliberadamente comprar productos por razones éticas, ambientales o económicas. La frecuencia de participación en cada una de las actividades de protesta se midió a través de una escala de valoración de 5 puntos que varía desde 1 (Nunca lo he hecho y nunca lo haría bajo ninguna circunstancia) hasta 5 (Lo he hecho muchas veces). El índice de comportamiento individual de protesta se calculó mediante la sumatoria de los puntajes reportados por ítem $(M=8.77 ; D T=4.12)$. La escala empleada cuenta con un nivel de consistencia interna satisfactorio ( $\alpha$ de Cronbach $=0.89$ ).

Uso político de redes sociales: Se consultaron 5 tipos de conductas políticas que pueden desarrollarse vía redes sociales. Centrando la atención en comportamientos de carácter informacional (recibir contenidos y noticias relacionadas con cuestiones políticas) y expresivo (escribir opiniones políticas; responder o comentar a los puntos de vista expresados por otros usuarios; compartir imágenes, links, vídeos y otros contenidos relacionados con la política o procesos electorales; chatear con otros sobre temas de carácter político o electoral). La frecuencia de participación en cada actividad se midió mediante una escala de valoración de 5 puntos que va desde 1 (Nunca) hasta 5 (Siempre). Las conductas señaladas se examinaron con respecto a las plataformas Facebooky Twitter. A partir de la sumatoria de las respuestas por ítem se cons- 
truyeron índices de uso político tanto para el caso de Facebook ( $M=8.42 ; D T=4.00 ; \alpha=0.89$ ) como Twitter ( $M=7.12 ; D T=3.61$; $\alpha=0.93)$, además de un índice agregado para las dos plataformas $(M=15.54 ; D T=6.95 ; \alpha=0.93)$.

Pertenencia a grupos políticos: Se evaluó el grado de involucramiento offline de los participantes con 9 tipos de agrupaciones de naturaleza política. Contemplándose partidos políticos, sindicatos, asociaciones profesionales, asociaciones de mujeres, organizaciones ambientalistas, grupos de defensa de los derechos animales, entre otras. La relación con cada grupo político se midió por medio de opciones de respuesta que varían desde 1 (Nunca he sido miembro) hasta 4 (Soy miembro y participo activamente). El índice de pertenencia a grupos políticos se obtuvo mediante la sumatoria de los puntajes asignados a cada ítem $(M=10.80 ; D T=3.82 ; \alpha=0.89)$.

Estatus socioeconómico: En primer lugar se obtuvo el ingreso neto equivalente de cada participante mediante la división de los ingresos familiares para el tamaño de hogar reportado (Castillo, Miranda y Cabib, 2013). Asumiendo al estatus socioeconómico como la combinación entre ingresos y escolaridad (Morris y Morris, 2013) se procedió a obtener el producto entre ingreso neto equivalente y nivel de educación, los valores resultantes se estandarizaron a puntajes- $\mathrm{z}(M=0 ; D T=1)$. Eficacia política: Para la dimensión externa se emplearon 4 ítems que evalúan la percepción individual sobre el grado de receptividad e interés del sistema político hacia las demandas y propuestas ciudadanas. En el caso de la dimensión interna se adaptó la escala de 4 ítems formulada por Niemi, Craig y Mattei (1991) para medir la competencia autopercibida por el individuo para participar en acciones políticas y lograr los objetivos propuestos (Zumárraga-Espinosa, 2020). En todos los casos las opciones de respuesta oscilaron entre 1 (Totalmente en desacuerdo) y 5 (Totalmente de acuerdo). Vía sumatoria se obtuvieron los respectivos índices de eficacia política externa $(M=10.96 ; D T=3.95 ; \alpha=0.85)$ e interna $(M=10.30$; $D T=3.42 ; \alpha=0.81$ ).

Emociones negativas: Se incluyeron 8 ítems para examinar respuestas emocionales negativas hacia la situación actual del país. Las emociones consultadas fueron: desprecio, odio, miedo, resentimiento, ansiedad, enfado, vergüenza y amargura. La escala de valoración utilizada va desde 1 ( Nada) hasta 5 (Extremadamente). Los valores se sumaron para calcular el respectivo índice aditivo $(M=16.94 ; D T=7.01 ; \alpha=0.88)$.

Situación ocupacional y características sociodemográficas: Se codificó con el valor de 1 a quienes reportaron no estar realizando labores remuneradas, el valor 0 se asignó al resto de casos. Asimismo se registró el género y la edad de los participantes.

Interés en la política: Con opciones de respuesta que oscilan entre 1 (Nada interesado) y 4 (Muy interesado) se realizó la siguiente pregunta, ¿Qué tan interesado diría Ud. que está en la política? ( $M=1.34 ; D T=0.91)$.

Ideología política: Partiendo de una escala que va desde 1 (Izquierda) hasta 10 (Derecha) se solicitó a los participantes seleccionar el valor que mejor represente su postura política $(M=5.28 ; D T=2.09)$.

Uso de medios de comunicación tradicionales: Se consultó la frecuencia con la que los participantes emplean medios tradicionales para informarse sobre asuntos políticos, de interés público o de actualidad. Se efectuaron mediciones para el caso de prensa escrita, televisión y radio. Las respuestas se reportaron a partir de una escala de valoración que varía desde 
1 (Nunca) hasta 5 (Siempre). Los puntajes asignados a cada medio se sumaron para la generación del índice agregado correspondiente $(M=7.05 ; D T=2.73 ; \alpha=0.83)$.

\section{Resultados}

La evaluación empírica de las hipótesis de investigación partió de la construcción de modelos de regresión multivariante, asignando el rol de variable dependiente a la participación en actividades offline de protesta política. La estimación de parámetros se efectuó por mínimos cuadrados ordinarios. El modelo 1 está compuesto por los siguientes predictores: uso político de redes sociales, estatus socioeconómico, pertenencia a grupos políticos, interacciones y el resto de variables de control consideradas. Cabe señalar que el índice de uso político de redes sociales integra las plataformas de Facebook y Twitter. Asimismo, para contrastar las relaciones de moderación propuestas en H2 y H3 se hace uso de 2 términos de interacción: uso políticos de redes sociales x estatus socioeconómico y uso político de redes sociales x pertenencia a grupos políticos.

Los resultados del análisis de regresión efectuado se muestran en la tabla 1. Como se puede observar, el uso político de redes sociales se relaciona de forma positiva con el comportamiento individual de protesta $(\beta=0.340, p<0.001)$, existiendo soporte empírico para H1. Entre los predictores de interés también están el estatus socioeconómico y la pertenencia a grupos políticos. En el caso del estatus socioeconómico se observa un efecto significativo y positivo sobre la participación en actividades de protesta $(\beta=0.133, \mathrm{p}<0.01)$, siendo las personas más educadas y con mayor disponibilidad de ingresos quienes tienen mayor predisposición a adoptar este tipo de conductas. Efecto similar, aunque de mayor magnitud, se presenta en relación con la pertenencia a grupos políticos $(\beta=0.162, \mathrm{p}<0.01)$. El estatus socioeconómico, la pertenencia a grupos políticos y el resto de variables de control explican el $28.6 \%$ de las variaciones del nivel de participación en acciones de protesta. Por su parte, el uso político de redes sociales contribuye con una varianza explicada de $12.5 \%$.

En cuanto al rol moderador del estatus socioeconómico y la pertenencia a grupos políticos, los términos de interacción introducidos en el análisis de regresión arrojan resultados estadísticamente significativos. Esto implica que el efecto del uso político de redes sociales sobre el comportamiento de protesta cambia significativamente ante variaciones en el estatus socioeconómico de los individuos $(\beta=-0.155 ; \mathrm{p}<0.01)$. Del mismo modo, esta relación entre conductas políticas vía redes sociales y protesta política se encuentra condicionada por el grado de pertenencia a grupos políticos $(\beta=0.202$; $\mathrm{p}<0.01$ ). A fin de analizar la naturaleza de las relaciones de moderación detectadas se procedió a realizar pruebas de pendiente simple, partiendo de las recomendaciones y recursos digitales proporcionados por Dawson (2014). La prueba de pendiente simple estima el efecto de un predictor sobre la variable dependiente según diferentes niveles de la variable moderadora. Para la selección de los valores condicionales del moderador se tomará como referencia el criterio "pick a point" de una desviación estándar por debajo $(-1 D E)$ y por arriba $(+1 D E)$ de la media (Preacher, Curran y Bauer, 2006). De esta manera es posible evaluar la relación de interés a valores relativamente altos y bajos del moderador. 
Tabla 1. Regresión multivariante del comportamiento de protesta política

\begin{tabular}{|c|c|}
\hline & $\begin{array}{c}\text { Modelo } 1 \\
\beta\end{array}$ \\
\hline \multicolumn{2}{|l|}{ Variables de control } \\
\hline Género (Masculino) & 0.034 \\
\hline Edad & $-0.057^{* *}$ \\
\hline Eficacia política externa & 0.031 \\
\hline Emociones negativas & $0.058^{* *}$ \\
\hline Situación ocupacional (No trabaja) & $0.044^{*}$ \\
\hline Interés en la política & -0.003 \\
\hline Ideología política & -0.011 \\
\hline Eficacia política interna & 0.009 \\
\hline Uso de medios de comunicación tradicionales & -0.041 \\
\hline Estatus socioeconómico & $0.133^{* *}$ \\
\hline Pertenencia a grupos políticos & $0.162^{* *}$ \\
\hline $\mathbf{R}^{2}$ & $28.6 \%$ \\
\hline \multicolumn{2}{|l|}{ Redes sociales } \\
\hline Uso político de redes sociales (Facebook y Twitter) & $0.340^{* * *}$ \\
\hline $\mathbf{R}^{2}$ Incremental & $12.5 \%$ \\
\hline \multicolumn{2}{|l|}{ Interacciones } \\
\hline Uso político de redes sociales x Pertenencia a grupos políticos & $0.202^{* *}$ \\
\hline Uso político de redes sociales x Estatus socioeconómico & $-0.155^{* *}$ \\
\hline $\mathbf{R}^{2}$ Incremental & $0.7 \%$ \\
\hline $\mathbf{R}^{2}$ Total & $41.8 \%$ \\
\hline Nota: ${ }^{*} \mathrm{p}<0.05(5 \%) ;{ }^{* *} \mathrm{p}<0.01(1 \%) ;{ }^{* * *} \mathrm{p}<0.001(0.1 \%)$. Se pres & esión estandarizados. \\
\hline
\end{tabular}

Con respecto a la posición socioeconómica, las pruebas efectuadas muestran un patrón de moderación negativo. Cuando el estatus socioeconómico adopta un valor relativamente bajo $(-1 D E)$, el efecto del uso político de redes sociales sobre el comportamiento de protesta es mayor $(\beta=0.239$; $p<0.001)$, en cambio dicho efecto disminuye $(\beta=0.162, p<0.001)$ en el caso de un nivel socioeconómico relativamente alto $(+1 D E)$. Por lo tanto, a medida que la educación y el nivel de ingresos de las personas sea menor, el uso político de redes sociales tiende a estimular con mayor fuerza la participación en actividades de protesta política (véase Figura 1). La relación entre uso político de redes sociales y protesta política es por lo general significativa, salvo que los valores $(z)$ del moderador (estatus socioeconómico) se sitúen dentro del intervalo que va desde 3.01 hasta 12.30. Para la estimación de la región de significación descrita se empleó los procedimientos y herramientas online facilitadas por Preacher et al. (2006), con un nivel de confianza del 95\%. 
Figura 1. Efecto moderador del estatus socioeconómico en la relación entre uso político de redes sociales y comportamiento de protesta

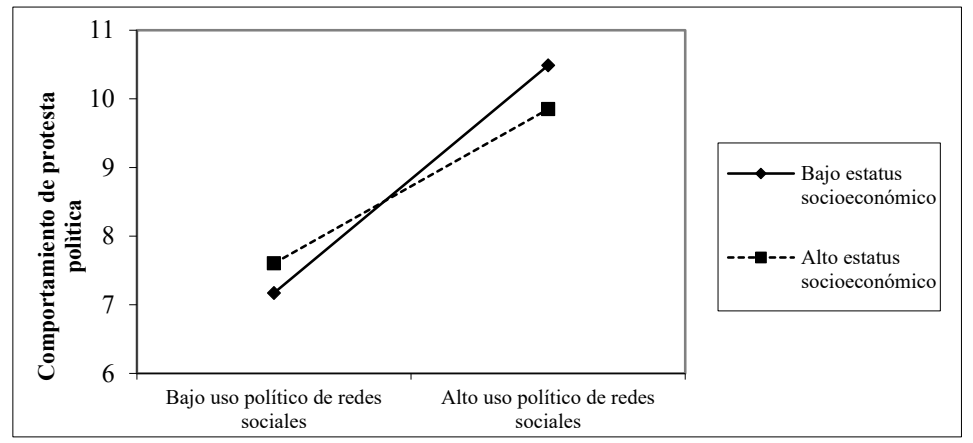

Fuente: Datos recolectados por el GIPS-UPS. Quito - Ecuador. Junio - Julio, 2018. Elaboración propia

El análisis de pendientes simples para la pertenencia a grupos políticos sugiere un patrón de moderación positivo. Cuando el moderador (pertenencia a grupos políticos) toma un valor relativamente alto $(+1 \mathrm{DE}$ ) la relación entre el uso político de redes sociales y participación en actos de protesta se intensifica $(\beta=0.277, \mathrm{p}<0.001)$, en cambio dicha relación se debilita $(\beta=0.237, \mathrm{p}<0.001)$ si el moderador adopta un valor relativamente bajo $(-1 D E)$. En consecuencia, el efecto movilizador del uso político de redes sociales sobre el comportamiento de protesta se fortalece a medida que los individuos se encuentren más involucrados con agrupaciones políticas en el ámbito offline (véase Figura 2). La relación entre uso político de redes sociales y protesta política es significativa para cualquier valor positivo del moderador examinado.

Figura 2. Efecto moderador de la pertenencia a grupos políticos en la relación entre uso político de redes sociales y comportamiento de protesta

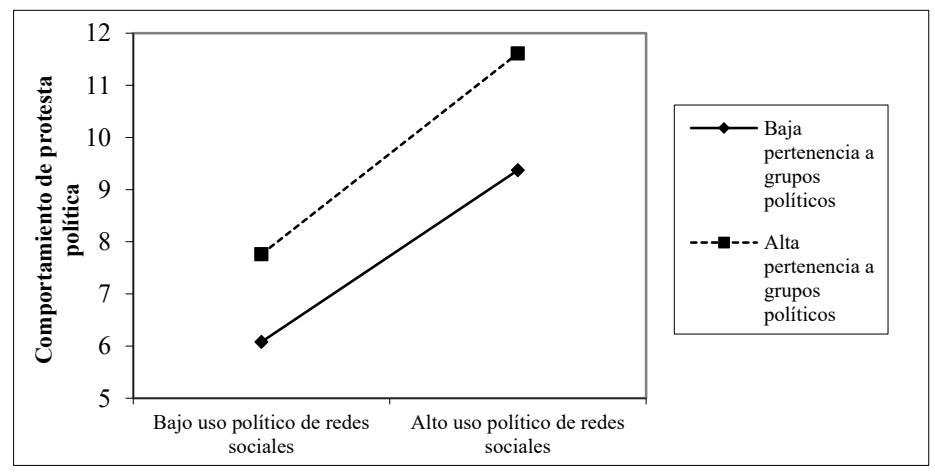

Fuente: Datos recolectados por el GIPS - UPS. Quito - Ecuador. Junio - Julio, 2018. Elaboración propia 
Redes sociales y protesta política: un análisis del rol moderador del estatus socioeconómico...

Tabla 2. Regresiones multivariantes del comportamiento de protesta política. Efectos desagregados para Facebook (modelo 2) y Twitter (modelo 3)

\begin{tabular}{|c|c|c|}
\hline & $\begin{array}{c}\text { Modelo } 2 \\
\beta\end{array}$ & $\begin{array}{c}\text { Modelo } 3 \\
\beta\end{array}$ \\
\hline \multicolumn{3}{|l|}{ Variables de control } \\
\hline Género (Masculino) & 0.040 & 0.039 \\
\hline Edad & $-0.081^{* * *}$ & $-0.068^{* *}$ \\
\hline Eficacia política externa & $0.050^{*}$ & 0.023 \\
\hline Emociones negativas & $0.071^{* *}$ & $0.074^{* * *}$ \\
\hline Situación ocupacional (No trabaja) & $0.044^{*}$ & $0.044^{*}$ \\
\hline Interés en la política & 0.003 & 0.021 \\
\hline Ideología política & -0.011 & -0.013 \\
\hline Eficacia política interna & 0.028 & 0.014 \\
\hline Uso de medios de comunicación tradicionales & -0.023 & -0.009 \\
\hline Estatus socioeconómico & 0.085 & $0.117^{* *}$ \\
\hline Pertenencia a grupos políticos & $0.191^{* * *}$ & $0.203^{* * *}$ \\
\hline $\mathbf{R}^{2}$ & $28.6 \%$ & $28.6 \%$ \\
\hline \multicolumn{3}{|l|}{ Redes sociales } \\
\hline Uso político de Facebook & $0.179^{* *}$ & \\
\hline Uso político de Twitter & & $0.346^{* * *}$ \\
\hline $\mathrm{R}^{2}$ Incremental & $7.3 \%$ & $12.4 \%$ \\
\hline \multicolumn{3}{|l|}{ Interacciones } \\
\hline Uso político de Facebook x Pertenencia a grupos políticos & $0.264^{* *}$ & \\
\hline Uso político de Facebook x Estatus socioeconómico & $-0.098^{*}$ & \\
\hline Uso político de Twitter $\mathrm{x}$ Pertenencia a grupos políticos & & $0.144^{*}$ \\
\hline Uso político de Twitter $\mathrm{x}$ Estatus socioeconómico & & $-0.145^{* *}$ \\
\hline $\mathbf{R}^{2}$ Incremental & $0.6 \%$ & $0.6 \%$ \\
\hline $\mathbf{R}^{2}$ Total & $36.5 \%$ & $41.6 \%$ \\
\hline
\end{tabular}

Nota: ${ }^{*} \mathrm{p}<0.05(5 \%) ;{ }^{* *} \mathrm{p}<0.01(1 \%) ;{ }^{* * *} \mathrm{p}<0.001(0.1 \%)$. Se presentan los coeficientes de regresión estandarizados.

Fuente: Datos recolectados por el GIPS - UPS. Quito - Ecuador. Junio - Julio, 2018. Elaboración propia 
Por último se efectuaron análisis de regresión específicos con el propósito de evaluar los efectos de Facebook (modelo 2) y Twitter (modelo 3) por separado. La tabla 2 presenta los resultados obtenidos. Si bien las dos plataformas influyen de manera significativa sobre el comportamiento de protesta al utilizarse políticamente, los coeficientes de regresión sugieren que Twitter ( $\beta=0.346, \mathrm{p}<0.001$ ) ejerce mayor efecto movilizador que Facebook $(\beta=0.179, \mathrm{p}<0.01)$. Por otra parte, los términos de interacción introducidos en cada modelo muestran que los patrones de moderación previamente identificados se conservan al desagregar el uso político de redes sociales por plataformas. El estatus socioeconómico produce un efecto moderador negativo sobre la relación entre el uso político de cada red social y la adopción de conductas de protesta. En tanto que el efecto moderador de la pertenencia a grupos políticos describe un patrón positivo, se trate de Facebook o Twitter. No obstante, el contraste realizado apunta a que el efecto moderador del estatus socioeconómico se intensifica en el caso de Twitter $(\beta=-0.145, \mathrm{p}<0.01)$, mientras que la pertenencia a grupos políticos modera con mayor fuerza el efecto movilizador del uso político de Facebook $(\beta=0.264$, p $<0.01$ ). En resumen, el conjunto de pruebas efectuadas permiten verificar $\mathrm{H} 2$ y $\mathrm{H} 3$.

\section{Discusión}

Estudios previos han realizado importantes avances en la identificación de las condiciones que hacen posible que el uso de redes sociales se conecte con la adopción de conductas de protesta a nivel individual. En esta línea, la indagación de variables mediadoras y moderadoras adquiere cada vez mayor relevancia. En cuanto a los mecanismos de mediación, la literatura especializada coincide al señalar que resulta más probable que las redes sociales estimulen comportamientos políticos offline cuando de por medio existe una utilización política de estas plataformas. Al respecto, los resultados obtenidos muestran que los usuarios que emplean sus redes sociales para actividades políticas como el consumo de información, la expresión de puntos de vista o la discusión interpersonal, tienden a participar con mayor frecuencia en acciones de protesta política. Replicándose, en este sentido, los hallazgos efectuados por investigaciones previas en torno a la relación entre el uso político de redes sociales y el comportamiento de protesta (Enjolras et al., 2013; Macafee y De Simone, 2012; Valenzuela, 2013; Valenzuela et al., 2012; 2016), además de aportar evidencia empírica más detallada con respecto a Facebook y Twitter.

Este trabajo también amplía la literatura referente a los factores que moderan la relación entre redes sociales y protesta política. En primera instancia, pudo observarse que el uso político de redes sociales moviliza con mayor fuerza la participación en actos de protesta para quienes pertenecen a estratos socioeconómicos más bajos, fenómeno que se intensifica para la plataforma Twitter. Esto sugiere que si bien las conductas informacionales y expresivas desplegadas vía redes sociales producen beneficios actitudinales y cognitivos que aumentan la predisposición para participar políticamente, estas ganancias difieren según el nivel socioeconómico de los usuarios. Ante esto, Morris y Morris (2013) esbozan una explicación para tales ganancias diferenciales basada en la tesis de la puerta de acceso de Baum (2003), proponiendo que las personas más educadas y con mayores ingresos tienden a estar más informadas e implicadas políticamente previo al contacto con las prestaciones políticas del internet, dada su mayor facilidad para involucrarse en actividades participativas e intervenir en procesos de comunicación política por diferentes vías offline. En otras palabras, las personas de 
mayor nivel socioeconómico cuentan con una predisposición elevada para comportarse políticamente que se explica en gran parte por su disponibilidad de recursos materiales, cognitivos y actitudinales. En tanto que el uso político de redes sociales desempeña un papel complementario, razón por la cual generaría un impacto más bien pequeño sobre dicha predisposición.

Por el contrario, dado que las personas de menor estatus socioeconómico tienden a encontrarse más desvinculadas del proceso político offline, el internet y las redes sociales suponen quizá la única vía de acceso para informarse, comunicarse y actuar políticamente, generando mayores beneficios a nivel cognitivo y motivacional. Beneficios que pasan a producir cambios más intensos en la propensión a intervenir en actividades participativas offline. En este orden de ideas, los hallazgos realizados proponen que las redes sociales ejercen un efecto democratizador sobre el activismo offline de tipo protesta, sumándose a resultados similares alcanzados por análisis previos enfocados en conductas políticas convencionales (Morris y Morris, 2013). Un rol democratizador que, por tanto, iría más allá de la movilización digital de quienes se encuentran al margen de la vida política, poniéndolos también en camino hacia formas de participación de mayor alcance en el mundo real.

Asimismo, la pertenencia a grupos políticos offline ejerce un efecto moderador sobre la relación entre uso político de redes sociales y las acciones de protesta. Es así que las conductas políticas expresivas e informacionales que tienen lugar en redes sociales producen mayor efecto sobre el comportamiento de protesta a medida que las personas tengan mayor vinculación con organizaciones políticas o movimientos sociales, fenómeno que ocurre con mayor fuerza al tratarse de Facebook. Partiendo de la revisión teórica efectuada inicialmente, el rol moderador observado en la pertenencia a grupos políticos puede explicarse a través de 3 mecanismos específicos. Primero, la lista de contactos en Twitter o Facebook de quienes pertenecen a agrupaciones políticas offline pasa a integrarse también por otros miembros de dichas organizaciones, configurando una audiencia con intereses y preocupaciones políticas similares. En estas condiciones, los actos expresivos cuentan con mayores oportunidades de retroalimentación, al tiempo que es más probable que las discusiones adquieran calidad deliberativa si tienen lugar entre usuarios que comparten membresía, debido al contacto previo cara a cara (Wright y Street, 2007). Esto terminaría reforzando los procesos mentales asociados con la expresión (Pingree, 2007) y la discusión política interpersonal (Eveland, 2004), que posteriormente conducen a la participación offline.

Segundo, la información consumida vía redes sociales posee mayor carga movilizadora para los miembros de grupos políticos, encontrándose más expuestos a recibir diferentes tipos de invitaciones para enrolarse en actividades políticas offline. Información movilizadora especialmente relevante para la gestación de acciones colectivas de protesta (Valenzuela, 2013), misma que puede provenir del propio grupo político al cual se pertenece, o de otras asociaciones afines con las que es posible interactuar mediante grupos online creados a través de las redes sociales. Tercero, la membresía a grupos políticos estimula la adopción de un rol más activo en el proceso de comunicación política. Por una parte, las discusiones online que tienen lugar entre miembros de un mismo grupo demandan que el resto de miembros exprese sus opiniones y puntos de vista, en especial si dicha agrupación cuenta con una versión online en redes sociales (Conroy et al., 2012). Además, el funcionamiento descentralizado que las organizaciones políticas y los movimientos sociales han adoptado gracias al internet y las redes sociales fomenta que sus miembros aporten más activamente en la promoción de 
los objetivos organizacionales, estando más propensos a expresar su apoyo y difundir información movilizadora en redes sociales, a la par de orientar esfuerzos de reclutamiento hacia a sus contactos digitales.

Finalmente, es pertinente señalar que este estudio presenta limitaciones asociadas con uso de datos transversales y la operacionalización de variables clave. En este sentido se requiere que futuras investigación confirmen, a partir de información longitudinal, la direccionalidad causal sugerida por los resultados aquí expuestos. Del mismo modo, se recomienda explorar con mayor profundidad otros usos políticos de redes sociales como la interacción con grupos políticos online, la utilización de memes como formas de humor político, entre otros. Siendo también necesario evaluar en qué medida las relaciones observadas se modifican al desagregar el análisis del comportamiento de protesta por actividades específicas como marchas no autorizadas, huelgas, boicots, plantones, bloqueo del tráfico, etc.

\section{Referencias bibliográficas}

Anduiza, E., Cristancho, C. y Sabucedo, J. (2014). Mobilization through online social networks: the political protest of the indignadosinSpain. Information,Communication \&Society,17(6),750-764.https:// doi.org/10.1080/1369118X.2013.808360 Anduiza, E., Gallego, A. y Cantijoch, M. (2010). Online political participation in Spain: the impact of traditional and Internet resources. Journal of Information Technology \& Politics, 7(4), 356-368. https://doi.org/10.1080/19331681003791891

Basabe-Serrano, S. y Martínez, J. (2014). Ecuador: Cada vez menos democracia, cada vez más autoritarismo... con elecciones. Revista de ciencia política, 34(1), 145-170. http://dx.doi.org/10.4067/S0718-090X2014000100007

Baum, M. (2003). Soft news goes to war: Public opinion and American foreign policy in the new media age. New Jersey: Princeton University Press.

Best, S. y Krueger, B. (2005). Analyzing the representativeness of Internet political participation. Political Behavior, 27(2), 183-216. https://doi.org/10.1007/s11109-005-3242-y

Bode, L. y Dalrymple, K. (2016). Politics in 140 characters or less: Campaign communication, network interaction, and political participation on Twitter. Journal of Political Marketing, 15(4), 311-332. https://doi.org/10.1080/15377857.2014 .959686

Boulianne, S. (2015). Social media use and participation: A meta-analysis of current research. Information, communication \& society 18(5): 524-538. https://doi.org/10.1080/1369118X.2015.1008542

Boyle, M. P.y Schmierbach, M. (2009). Media use and protest:The role of mainstream and alternative media use in predicting traditional and protest participation. Communication Quarterly, 57(1), 1-17. https://doi.org/10.1080/01463370802662424

Brady, H., Verba, S. y Schlozman, K. (1995). Beyond SES: A resource model of political participation. American political science review, 89(2), 271-294. https://doi.org/10.2307/2082425

Calenda, D. y Meijer, A. (2009). Young people, the Internet and political participation: findings of a web survey in Italy, Spain and The Netherlands. Information, Communication \& Society, 12(6), 879-898. https://doi.org/10.1080/13691180802158508 
Campbell, S. y Kwak, N. (2011). Political involvement in "mobilized" society: The interactive relationships among mobile communication, network characteristics, and political participation. Journal of Communication, 61(6), 1005-1024. https://doi.org/10.1111/j.1460-2466.2011.01601.x

Carlisle, J. y Patton, R. (2013). Is social media changing how we understand political engagement? An analysis of Facebook and the 2008 presidential election. Political Research Quarterly, 66(4), 883-895. https://doi.org/10.1177/1065912913482758

Carty, V. (2010). New information communication technologies and grassroots mobilization. Information, Communication \& Society, 13(2), 155-173. https://doi.org/10.1080/13691180902915658

Chan, M. (2016). Social network sites and political engagement: Exploring the impact of Facebook connections and uses on political protest and participation. Mass communication and society, 19(4), 430-451. https://doi.org/10.1080/1520543 6.2016.1161803

Cho, J., Shah, D., McLeod, J., McLeod, D., Scholl, R. y Gotlieb, M. (2009). Campaigns, reflection, and deliberation: Advancing an OSROR model of communication effects. Communication Theory, 19(1), 66-88. https://doi. org/10.1111/j.1468-2885.2008.01333.x

Choi, J. (2016). Differential use, differential effects: Investigating the roles of different modes of news use in promoting political participation. Journal ofComputer-Mediated Communication, 21(6), 436-450. https://doi.org/10.1111/jcc4.12176

Conroy, M., Feezell, J. y Guerrero, M. (2012). Facebook and political engagement: A study of online political group membership and offline political engagement. Computers in Human behavior, 28(5), 1535-1546. https://doi.org/10.1016/j. chb.2012.03.012

Cristancho, C. y Anduiza, E. (2013). Connective Action in European Mass Protest. Social Media and Political Participation Conference, La Pietra Dialogues, NYU. http://www.lapietradialogues.org/area/pubblicazioni/doc000078.pdf

Dalton, R. (2008). Citizenship norms and the expansion of political participation. Political studies, 56(1), 76-98. https:// doi.org/10.1111/j.1467-9248.2007.00718.x

Dalton, R., Van Sickle, A. y Weldon, S. (2010). The individual-institutional nexus of protest behavior. British Journal of Political Science 40(1): 51-73. https://doi.org/10.1017/S000712340999038X

Dawson, J. (2014). Moderation in management research: What, why, when, and how. Journal of Business and Psychology, 29(1), 1-19. https://doi.org/10.1007/s10869-013-9308-7

DiGrazia, J. (2014). Individual protest participation in the United States: Conventional and unconventional activism. Social Science Quarterly, 95(1), 111-131. https://doi.org/10.1111/ssqu.12048

Dubrow, J., Slomczynski, K. y Tomescu-Dubrow, I. (2008). Effects of democracy and inequality on soft political protest in Europe: exploring the European social survey data. International Journal of Sociology, 38(3), 36-51. https://doi. org/10.2753/IJS0020-7659380302

Ekman, J. y Amnå, E. (2012). Political participation and civic engagement: Towards a new typology. Human affairs, 22(3), 283-300. https://doi.org/10.2478/s13374-012-0024-1 
Enjolras, B., Steen-Johnsen, K. y Wollebæk, D. (2013). Social media and mobilization to offline demonstrations: Transcending participatory divides?. New media \& society, 15(6), 890-908. https://doi.org/10.1177/1461444812462844

Eveland, W. (2001). The cognitive mediation model of learning from the news: Evidence from nonelection, off-year election, and presidential election contexts. Communication Research,28(5),571-601.https://doi.org/10.1177/009365001028005001

Eveland, W. (2004). The effect of political discussion in producing informed citizens: The roles of information, motivation, and elaboration. Political Communication, 21(2), 177-193. https://doi.org/10.1080/10584600490443877

Gibson, R. (2015). Party change, social media and the rise of 'citizen-initiated'campaigning. Party politics, 21(2), 183-197. https://doi.org/10.1177/1354068812472575

Gil de Zúñiga, H., Ardèvol-Abreu, A. y Casero-Ripollés, A. (2019). WhatsApp political discussion, conventional participation and activism: exploring direct, indirect and generational effects. Information, Communication \& Society, 1-18. https://doi. org/10.1080/1369118X.2019.1642933

Gil de Zúñiga, H., Jung, N. y Valenzuela, S. (2012). Social media use for news and individuals' social capital, civic engagement and political participation. Journal of computer-mediated communication, 17(3), 319-336. https://doi. org/10.1111/j.1083-6101.2012.01574.x

Gil de Zúñiga, H., Molyneux, L. y Zheng, P. (2014). Social media, political expression, and political participation: Panel analysis of lagged and concurrent relationships. Journal of Communication, 64(4), 612-634. https://doi.org/10.1111/ jcom.12103

Gil de Zúñiga, H., Veenstra, A., Vraga, E. y Shah, D. (2010). Digital democracy: Reimagining pathways to political participation. Journal of Information Technology \& Politics, 7(1), 36-51. https://doi.org/10.1080/19331680903316742

Gustafsson, N. (2012). The subtle nature of Facebook politics: Swedish social network site users and political participation. New Media \& Society, 14(7), 1111-1127. https://doi.org/10.1177/1461444812439551

Hardy, B. y Scheufele, D. (2005). Examining differential gains from Internet use: Comparing the moderating role of talk and online interactions. Journal of Communication, 55(1), 71-84. https://doi.org/10.1111/j.1460-2466.2005.tb02659.x

Heiss, R. y Matthes, J. (2016). Mobilizing for Some: The Effects of Politicians' Participatory Facebook Posts on Young People's Political Efficacy. Journal of Media Psychology, 28 (3), 123-135. https://doi.org/10.1027/1864-1105/a000199

Ji, Y., Zhou, Y. y Kim, S. (2017). A moderated mediation model of political collective action in Hong Kong: examining the roles of social media consumption and social identity. Asian Journal of Communication, 27(5), 497-516. https://doi.org/ 10.1080/01292986.2017.1336777

Jung, N., Kim, Y. y Gil de Zúñiga, H. (2011). The mediating role of knowledge and efficacy in the effects of communication on political participation. Mass Communication and Society, 14(4), 407-430. https://doi.org/10.1080/15205436.2010.496 135

Kaye, B. y Johnson, T. (2002). Online and in the know: Uses and gratifications of the web for political information. Journal of Broadcasting \& Electronic Media, 46(1), 54-71. https://doi.org/10.1207/s15506878jobem4601_4 
Kim, Y., Chen, H. T. y Gil de Zúñiga, H. (2013). Stumbling upon news on the Internet: Effects of incidental news exposure and relative entertainment use on political engagement. Computers in human behavior, 29(6), 2607-2614. https://doi. org/10.1016/j.chb.2013.06.005

Kim, Y., Hsu, S. y Gil de Zúñiga, H. (2013). Influence of social media use on discussion network heterogeneity and civic engagement:The moderating role of personality traits. Journal ofCommunication, 63(3), 498-516. https://doi.org/10.1111/ jcom.12034

Krueger, B. (2006). A comparison of conventional and Internet political mobilization. American Politics Research, 34(6), 759-776. https://doi.org/10.1177/1532673X06290911

Krueger, B. (2002). Assessing the potential of Internet political participation in the United States: A resource approach. American Politics Research, 30(5), 476-498. https://doi.org/10.1177/1532673X02030005002

Lee, F., Chen, H. T. y Chan, M. (2017). Social media use and university students' participation in a large-scale protest campaign: The case of Hong Kong's Umbrella Movement. Telematics and Informatics, 34(2), 457-469. https://doi. org/10.1016/j.tele.2016.08.005

Lee, S. (2019). Connecting social media use with gaps in knowledge and participation in a protest context: the case of candle light vigil in South Korea. Asian Journal of Communication, 29(2), 111-127. https://doi.org/10.1080/01292986.201 8.1549264

Lemert, J. B. (1981). Does mass communication change public opinion after all? A new approach to effects analysis. Chicago, IL: Nelson-Hall.

Lenzi, M., Vieno, A., Altoè, G., Scacchi, L., Perkins, D., Zukauskiene, R. y Santinello, M. (2015). Can Facebook informational use foster adolescent civic engagement?. American Journal of Community Psychology, 55(3-4), 444-454. https://doi. org/10.1007/s10464-015-9723-1

Macafee, T. y De Simone, J.J. (2012). Killing the bill online? Pathways to young people's protest engagement via social media. Cyberpsychology, Behavior, and Social Networking, 15(11), 579-584. https://doi.org/10.1089/cyber.2012.0153

Meléndez, C. y Moncagatta, P. (2017). Ecuador: Una década de correísmo. Revista de ciencia política, 37(2), 413-448. http:// dx.doi.org/10.4067/s0718-090x2017000200413

Micó, J.L y Casero-Ripollés, A. (2014). Political activism online: organization and media relations in the case of 15M in Spain. Information, Communication \& Society, 17(7), 858-871. https://doi.org/10.1080/1369118X.2013.830634

Morris, D. y Morris, J. (2013). Digital inequality and participation in the political process: Real or imagined?. Social Science Computer Review, 31(5), 589-600. https://doi.org/10.1177/0894439313489259

Mutz, D. (2002). Cross-cutting social networks: Testing democratic theory in practice. American Political Science Review, 96(1), 111-126. https://doi.org/10.1017/S0003055402004264

Nam, T. (2012). Dual effects of the internet on political activism: Reinforcing and mobilizing. Government Information Quarterly, 29, S90-S97. https://doi.org/10.1016/j.giq.2011.08.010 
Niemi, R., Craig, S. y Mattei, F. (1991). Measuring internal political efficacy in the 1988 National Election Study. American Political Science Review, 85(4), 1407-1413. https://doi.org/10.2307/1963953

Norris, P., Walgrave, S. y Van Aelst, P. (2005). Who demonstrates? Antistate rebels, conventional participants, or everyone?. Comparative politics, 37(2), 189-205.

Oser, J., Hooghe, M. y Marien, S. (2013). Is online participation distinct from offline participation? A latent class analysis of participation types and their stratification. Political Research Quarterly, 66(1), 91-101. https://doi. org/10.1177/1065912912436695

Pingree, R. (2007). How messages affect their senders: A more general model of message effects and implications for deliberation. Communication Theory, 17(4), 439-461. https://doi.org/10.1111/j.1468-2885.2007.00306.x

Preacher, K., Curran, P. y Bauer, D. (2006). Computational tools for probing interactions in multiple linear regression, multilevel modeling, and latent curve analysis. Journal of educational and behavioral statistics, 31(4), 437-448. https:// doi.org/10.3102/10769986031004437

Putnam, R. (2000). Bowling alone: The collapse and revival of American community. New York: Simon \& Schuster.

Rojas, H.yPuig-i-Abril,E. (2009). Mobilizers mobilized:Information, expression, mobilization and participation in the digital age. Journal of Computer-Mediated Communication, 14(4), 902-927. https://doi.org/10.1111/j.1083-6101.2009.01475.x

Scherman, A., Arriagada, A. y Valenzuela, S. (2015). Student and environmental protests in Chile: The role of social media. Politics, 35(2), 151-171. https://doi.org/10.1111/1467-9256.12072

Schlozman, K., Verba, S. y Brady, H. (2010). Weapon of the strong? Participatory inequality and the Internet. Perspectives on Politics, 8(2), 487-509. https://doi.org/10.1017/S1537592710001210

Schussman, A. y Soule, S. (2005). Process and protest: Accounting for individual protest participation. Social forces, 84(2), 1083-1108. https://doi.org/10.1353/sof.2006.0034

Scheufele, D. y Nisbet, M. (2002). Being a citizen online: New opportunities and dead ends. Harvard International Journal of Press/Politics, 7(3), 55-75. https://doi.org/10.1177/1081180X0200700304

Shah, D., Cho, J., Eveland, W. y Kwak, N. (2005). Information and expression in a digital age: Modeling Internet effects on civic participation. Communication research, 32(5), 531-565. https://doi.org/10.1177/0093650205279209

Shah, D., Cho, J., Nah, S., Gotlieb, M., Hwang, H., Lee, N.J., Scholl, R. y McLeod. D. (2007). Campaign ads, online messaging, and participation: Extending the communication mediation model. Journal of communication, 57(4), 676-703. https:// doi.org/10.1111/j.1460-2466.2007.00363.x

Tang, G. y Lee, F. (2013). Facebook use and political participation: The impact of exposure to shared political information, connections with public political actors, and network structural heterogeneity. Social Science Computer Review, 31(6), 763-773. https://doi.org/10.1177/0894439313490625 
Theocharis, Y., Lowe, W., Van Deth, J. y García-Albacete, G. (2015). Using Twitter to mobilize protest action: online mobilization patterns and action repertoires in the Occupy Wall Street, Indignados, and Aganaktismenoi movements. Information, Communication \& Society, 18(2), 202-220. https://doi.org/10.1080/1369118X.2014.948035

Valenzuela, S. (2013). Unpacking the use of social media for protest behavior:The roles of information, opinion expression, and activism. American Behavioral Scientist, 57(7), 920-942. https://doi.org/10.1177/0002764213479375

Valenzuela, S., Arriagada, A. y Scherman, A. (2012). The social media basis of youth protest behavior: The case of Chile. Journal of Communication, 62(2), 299-314. https://doi.org/10.1111/j.1460-2466.2012.01635.x

Valenzuela, S., Park, N. y Kee, K. (2009). Is there social capital in a social network site?: Facebook use and college students' life satisfaction, trust, and participation. Journal of computer-mediated communication 14(4): 875-901. https://doi. org/10.1111/j.1083-6101.2009.01474.x

Valenzuela, S., Somma, N., Scherman, A. y Arriagada, A. (2016). Social media in Latin America: deepening or bridging gaps in protest participation?. Online Information Review, 40(5), 695-711.

Valeriani, A. yVaccari, C. (2016). Accidental exposure to politics on social media as online participation equalizer in Germany, Italy, and the United Kingdom. New Media \& Society, 18(9), 1857-1874. https://doi.org/10.1177/1461444815616223

Verba, S., Schlozman, K. y Brady, H. (1995). Voice and equality: Civic voluntarism in American politics. London: Harvard University Press.

Wang, S.I. (2007). Political use of the Internet, political attitudes and political participation. Asian JournalofCommunication, 17(4), 381-395. https://doi.org/10.1080/01292980701636993

Winneg, K. (2009). Online political participation in the 2008 US presidential election: Mobilizing or reinforcing?. Publicly accessible Penn Dissertations 72.

Wright, S. y Street, J. (2007). Democracy, deliberation and design: the case of online discussion forums. New media \& society, 9(5), 849-869. https://doi.org/10.1177/1461444807081230

Xenos, M., Vromen, A. y Loader, B. (2014). The great equalizer? Patterns of social media use and youth political engagement in three advanced democracies. Information, Communication \& Society, 17(2), 151-167. https://doi. org/10.1080/1369118X.2013.871318

Yamamoto, M., Kushin, M. y Dalisay, F. (2015). Social media and mobiles as political mobilization forces for young adults: Examining the moderating role of online political expression in political participation. New Media \& Society, 17(6), 880898. https://doi.org/10.1177/1461444813518390

Yang, H. y DeHart, J. (2016). Social media use and online political participation among college students during the US election 2012. Social Media+ Society, 2(1), 1-18. https://doi.org/10.1177/2056305115623802

Yoo, S.W. y Gil de Zúñiga, H. (2019). The role of heterogeneous political discussion and partisanship on the effects of incidental news exposure online. Journal of Information Technology \& Politics, 1-16. https://doi.org/10.1080/19331681.2 018.1561346

76 | n 30, pp. 55-77 | doxa.comunicación

enero-junio de 2020 
Yoo, S.W. y Gil de Zúñiga, H. (2014). Connecting blog, Twitter and Facebook use with gaps in knowledge and participation. Communication \& Society, 27 (4), 33-48.

Zhang, W. y Chia, S. (2006). The effects of mass media use and social capital on civic and political participation. Communication Studies, 57(3), 277-297. https://doi.org/10.1080/10510970600666974

Zumárraga-Espinosa, M. (2020). Aportes metodológicos para la medición del sentido de eficacia politica: Evidencia empírica de Quito-Ecuador. Empiria. Revista de metodología de ciencias sociales, (45), 113-142. https://doi.org/10.5944/ empiria.45.2020.26306

Zumárraga-Espinosa, M., Carofilis-Cedeño, C. y Reyes-Valenzuela, C. (2017). El efecto movilizador de las emociones sobre la participación política online en la segunda vuelta de las elecciones presidenciales en Ecuador 2017. Revista Democracias, 5, 25-62.

Zumárraga-Espinosa, M., Reyes-Valenzuela, C. y Carofilis-Cedeño, C. (2017). ¿ Verdad o ficción? El uso político de las redes sociales en la participación política offline en las elecciones presidenciales en Ecuador. Análisis político, 30(91), 130-145. https://doi.org/10.15446/anpol.v30n91.70268 